\title{
HADAMARD'S INEQUALITY ON A TRIANGLE AND ON A POLYGON
}

\author{
YIN CHEN
}

\begin{abstract}
In this paper, some inequalities of Hadamard's type for a convex function of double variable defined on a triangle and on a regular polygon are proved. As a consequence, Hadamard's inequality on a disk is also given.
\end{abstract}

\section{Introduction}

Let $f$ be a convex function defined on $[a, b]$. Then the following double inequality

$$
f\left(\frac{a+b}{2}\right) \leq \frac{1}{b-a} \int_{a}^{b} f(x) d x \leq \frac{f(a)+f(b)}{2}
$$

is called in the literature Hadamard's inequality.

Let us have a closer look at the second inequality. It states in fact that the average of a convex function on $[a, b]$ is less than or equal to the average of its values at the end points of $[a, b]$.

We now consider a convex function on a triangle of $\mathbf{R}^{2}$. It is natural to ask if the average of the convex function on the triangle is less than or equal to the average of all the values of the function at the vertices. Recall that a function $f$ defined on a convex planar domain $D$ is said to be convex if it satisfies the following inequality

$$
f\left(\lambda_{1} x_{1}+\lambda_{2} x_{2}, \lambda_{1} y_{1}+\lambda_{2} y_{2}\right) \leq \lambda_{1} f\left(x_{1}, y_{1}\right)+\lambda_{2} f\left(x_{2}, y_{2}\right)
$$

for any $\left(x_{1}, y_{1}\right),\left(x_{2}, y_{2}\right) \in D$ and for any nonnegative constants $\lambda_{1}$ and $\lambda_{2}$ where $\lambda_{1}+\lambda_{2}=$ 1. Note that a convex function $f(x, y)$ of double variables is convex in both $x$ and $y$.

We will show that in fact the second inequality of (1) is also true for a convex function on a triangle, but not for a polygon in general.

\section{Hadamard's Inequality on a Triangle}

To establish the Hadamard's inequality on a triangle, we first need some lemmas. The following easy lemma shows that the composite function of a convex function and an affine transformation is also convex.

Received Feburary 25, 2003.

2000 Mathematics Subject Classification. 26D15, 26D07.

Key words and phrases. Hadamard's inequality, convex function of double variables, Jensen's inequality, regular polygon. 
Lemma 2.1. If $f(x, y)$ is a convex function on $D$, then the function $f(a x+b y+$ $m, c x+d y+n)$ is convex on $D^{\prime}$, where $D$ and $D^{\prime}$ are convex domains such that $D=$ $\left\{(a x+b y+m, c x+d y+n):(x, y) \in D^{\prime}\right\}$.

Proof. Directly verify the function $f(a x+b y+m, c x+d y+n)$ according to the definition of two-dimensional convexity of function.

The next lemma is just a special case of Theorem 2.4.

Lemma 2.2. Let $f(x, y)$ be a convex function on the triangle $\Delta_{0}$ enclosed by $x=0$, $y=0$ and $x+y=1$. Then

$$
2 \iint_{\Delta_{0}} f(x, y) d x d y \leq \frac{f(0,0)+f(0,1)+f(1,0)}{3} .
$$

Proof. $f(x, y)$ being convex function on $\Delta_{0}$ implies that the following inequalities hold for any $x$ between 0 and 1

$$
f(x, 0) \leq x f(1,0)+(1-x) f(0,0)
$$

and

$$
f(x, 1-x) \leq x f(1,0)+(1-x) f(0,1) .
$$

Since $f$ is convex on $y$, it follows from (1) that

$$
\int_{0}^{1-x} f(x, y) d y \leq(1-x) \cdot \frac{f(x, 0)+f(x, 1-x)}{2} .
$$

Therefore by (3), (4) and (5), we have

$$
\begin{aligned}
2 \iint_{\Delta_{0}} f(x, y) d x d y & =2 \int_{0}^{1} \int_{0}^{1-x} f(x, y) d y d x \\
& \leq \int_{0}^{1}(1-x)[f(x, 0)+f(x, 1-x)] d x \\
& \leq \int_{0}^{1}\left[2(1-x) x f(1,0)+(1-x)^{2} f(0,0)+(1-x)^{2} f(0,1)\right] d x \\
& \leq \frac{f(0,0)+f(0,1)+f(1,0)}{3} .
\end{aligned}
$$

This proves Lemma 2.2 .

The following lemma gives a formula for calculating the area of a triangle if the equations of its three sides are given. 
Lemma 2.3. Let $\Delta$ be a triangle enclosed by the following three lines

$$
l_{i}: c_{i} x+d_{i} y-m_{i}=0 \quad \text { for } i=1,2,3 .
$$

Then the area $A(\Delta)$ of the triangle $\Delta$ is given by

$$
A(\Delta)=\frac{1}{2} \frac{\left|\begin{array}{lll}
c_{1} & d_{1} & m_{1} \\
c_{2} & d_{2} & m_{2} \\
c_{3} & d_{3} & m_{3}
\end{array}\right|^{2}}{\| \begin{array}{ll}
c_{1} & d_{1} \\
c_{2} & d_{2}
\end{array}|\cdot| \begin{array}{ll}
c_{2} & d_{2} \\
c_{3} & d_{3}
\end{array}|\cdot| \begin{array}{ll}
c_{3} & d_{3} \\
c_{1} & d_{1}
\end{array}||} .
$$

Proof. We first change the variables $x, y$ to $X, Y$ by an affine transformation

$$
\begin{aligned}
& X=k\left(c_{1} x+d_{1} y-m_{1}\right), \\
& Y=l\left(c_{2} x+d_{2} y-m_{2}\right)
\end{aligned}
$$

where $k, l$ are two constants to be determined such that the equation

$$
X+Y-1=s\left(c_{3} x+d_{3} y-m_{3}\right)
$$

holds for some constant $s \neq 0$. In fact, an elementary calculation shows that

$$
k=\frac{c_{3} d_{2}-c_{2} d_{3}}{A}, \quad l=\frac{c_{1} d_{3}-c_{3} d_{1}}{A} \quad \text { and } \quad s=\frac{c_{1} d_{2}-c_{2} d_{1}}{A}
$$

where

$$
A=\left|\begin{array}{lll}
c_{1} & d_{1} & m_{1} \\
c_{2} & d_{2} & m_{2} \\
c_{3} & d_{3} & m_{3}
\end{array}\right|
$$

It is clear that $A \neq 0$, since otherwise all the three lines meet at the same point. Under this affine transformation, $l_{1}, l_{2}$ and $l_{3}$ become $X=0, Y=0$ and $X+Y=1$ respectively by $(7),(8)$ and $(9)$, hence $\Delta$ becomes now $\Delta_{0}$. It is easy to find the jacobian of this transformation

$$
|J|=\left|\frac{\partial(x, y)}{\partial(X, Y)}\right|=\frac{A^{2}}{\left|c_{1} d_{2}-c_{2} d_{1}\right| \cdot\left|c_{2} d_{3}-c_{3} d_{2}\right| \cdot\left|c_{3} d_{1}-c_{1} d_{3}\right|} .
$$

It follows that

$$
\begin{aligned}
A(\Delta) & =\iint_{\Delta} d x d y \\
& =\iint_{\Delta_{0}}|J| d X d Y \\
& =|J| \int_{0}^{1} \int_{0}^{1-X} d Y d X \\
& =\frac{1}{2}|J|,
\end{aligned}
$$


which is indeed (6).

We are now ready to give the Hadamard's inequality on a triangle.

Theorem 2.4. Let $f(x, y)$ be a convex function on a triangle $\Delta$ with the vertices $\left(a_{1}, b_{1}\right),\left(a_{2}, b_{2}\right)$ and $\left(a_{3}, b_{3}\right)$. Then

$$
\frac{1}{A(\Delta)} \iint_{\Delta} f(x, y) d x d y \leq \frac{f\left(a_{1}, b_{1}\right)+f\left(a_{2}, b_{2}\right)+f\left(a_{3}, b_{3}\right)}{3},
$$

where $A(\Delta)$ is the area of the triangle $\Delta$.

Proof. Suppose that $c_{i} x+d_{i} y-m_{i}=0(i=1,2,3)$ is the equation of the side line $l_{i}$ of the triangle $\Delta$ which does not meet the vertex $\left(a_{i}, b_{i}\right)$. We do an affine transformation as in the proof of Lemma 2.3 to change the variables $x, y$ to $X, Y$. Under this change of variable, $l_{1}, l_{2}$ and $l_{3}$ become $X=0, Y=0$ and $X+Y=1$ respectively. The three vertices become now $(1,0),(0,1)$ and $(0,0)$.

Set

$$
F(X, Y)=f\left(\frac{\left(\frac{X}{k}+m_{1}\right) d_{2}-\left(\frac{Y}{l}+m_{2}\right) d_{1}}{c_{1} d_{2}-c_{2} d_{1}}, \frac{-\left(\frac{X}{k}+m_{1}\right) c_{2}+\left(\frac{Y}{l}+m_{2}\right) c_{1}}{c_{1} d_{2}-c_{2} d_{1}}\right) .
$$

It follows by Lemma 2.1 that $F(X, Y)$ is convex on $\Delta_{0}$ since $f(x, y)$ is covnex on $\Delta$. Hence by Lemma 2.3 and then by Lemma 2.2 , we have

$$
\begin{aligned}
\frac{1}{A(\Delta)} \iint_{\Delta} f(x, y) d x d y & =\frac{2}{|J|} \iint_{\Delta_{0}} F(X, Y)|J| d X d Y \\
& =2 \iint_{\Delta_{0}} F(X, Y) d X d Y \\
& \leq \frac{F(0,0)+F(1,0)+F(0,1)}{3} \\
& =\frac{f\left(a_{1}, b_{1}\right)+f\left(a_{2}, b_{2}\right)+f\left(a_{3}, b_{3}\right)}{3} .
\end{aligned}
$$

This completes the proof.

\section{Hadamard's Inequality on a Polygon}

In Section 2, we show that the average of a convex function on an arbitrary triangle is less than or equal to the average of its values at the vertices. Is this true for a convex function on a convex polygon? Unfortunately, it is quite not the case in general.

Let us consider an example. Let $D$ be a quadrilateral enclosed by $x=0, y=0, y=1$ and $x+y=2$, and let $f(x, y)=x$. Then $f$ is convex on $D$. But it is easy to show 
that $\frac{1}{A(D)} \iint_{D} f(x, y) d x d y=\frac{7}{9}$ which is greater than the average of the values at all four vertices $\frac{3}{4}$.

The above example shows that even for a convex quadrilateral, Hadamard's inequality does not hold in general. However for the more regular quadrilaterals, we do have the Hadamard's inequality.

Theorem 3.1. Let $D$ be a parallelogram and $f(x, y)$ be convex on $D$, then

$$
f(a, b) \leq \frac{1}{A(D)} \iint_{D} f(x, y) d x d y \leq \frac{\sum_{i=1}^{4} f\left(a_{i}, b_{i}\right)}{4}
$$

where $(a, b)$ is the centre of $D,\left(a_{i}, b_{i}\right)(i=1,2,3,4)$ are the vertices and $A(D)$ is the area of $D$.

Proof. Without loss of generality, we can assume that $a=0, b=0$. Since a parallelogram is symmetric about its centre, letting $u=-x, v=-y$, we will have

$$
\iint_{D} f(x, y) d x d y=\iint_{D} f(-u,-v) d u d v=\iint_{D} f(-x,-y) d x d y .
$$

Hence

$$
\begin{aligned}
\iint_{D} f(x, y) d x d y & =\frac{1}{2}\left[\iint_{D} f(x, y) d x d y+\iint_{D} f(-x,-y) d x d y\right] \\
& =\iint_{D} \frac{1}{2}[f(x, y)+f(-x,-y)] d x d y \\
& \geq \iint_{D} f(0,0) d x d y=f(0,0) A(D) .
\end{aligned}
$$

This proves the first inequality of (12).

To prove the second inequality, let us first split the parallelogram into two triangles with the same area by one diagonal. On each triangle, make use of Theorem 2.4, then add the two integrals. We have thus $\frac{2}{A(D)} \iint_{D} f(x, y)$ is less than or equal to a sum of six terms of the values at the vertices over 3. Do the same about the other diagonal, then we have $\frac{2}{A(D)} \iint_{D} f(x, y)$ is less than or equal to a sum of six terms (they may be different from the previous six terms!) of the values at the vertices over 3 . Now add both sides of these two inequalities. The left side now becomes $\frac{4}{A(D)} \iint_{D} f(x, y) d x d y$ and the right side is a sum of 12 terms of values over 3 . Note that in the right side, the value of $f$ at each vertex appears exactly three times, thus the right side becomes the sum of the values of $f$ at all the four vertices. Dividing both sides by 4 gives the second part of (12). 
We will now extend (12) to a regular polygon. First we need the following Jensen's inequality.

Lemma 3.2. If $f(x, y)$ is convex on a convex domain $D$, then for any positive integer $n$ and any points $\left(x_{1}, y_{1}\right),\left(x_{2}, y_{2}\right), \ldots,\left(x_{n}, y_{n}\right) \in D$, we have

$$
f\left(\frac{\sum_{k=1}^{n} x_{k}}{n}, \frac{\sum_{k=1}^{n} y_{k}}{n}\right) \leq \sum_{k=1}^{n} \frac{f\left(x_{k}, y_{k}\right)}{n}
$$

Proof. Use mathematical induction. See also ([3], page 89).

The following lemma is about two easy trigonometric identities. They can be proved also by Euler's formula.

Lemma 3.3. If $n$ is any positive integer greater than 1 , then

$$
\sum_{k=0}^{n-1} \cos \frac{2 k \pi}{n}=\sum_{k=0}^{n-1} \sin \frac{2 k \pi}{n}=0 .
$$

Proof. Note that

$$
\begin{aligned}
\sin \frac{\pi}{n} \sum_{k=0}^{n-1} \cos \frac{2 k \pi}{n} & =\sum_{k=0}^{n-1} \sin \frac{\pi}{n} \cdot \cos \frac{2 k \pi}{n} \\
& =\sum_{k=0}^{n-1}\left(\sin \frac{(2 k+1) \pi}{n}-\sin \frac{(2 k-1) \pi}{n}\right) \\
& =\sin \frac{(2 n-1) \pi}{n}-\sin \frac{(-\pi)}{n}=0
\end{aligned}
$$

It follows that $\sum_{k=0}^{n-1} \cos \frac{2 k \pi}{n}=0$, since $\sin \frac{\pi}{n} \neq 0$.

Similarly $\sum_{k=0}^{n-1} \sin \frac{2 k \pi}{n}=0$.

We now give the Hadamard's inequality on a regular polygon.

Theorem 3.4. Let $n$ be a positive integer greater than 2 . Let $D$ be a regular $n$-gon and $f(x, y)$ be a convex function on $D$. Then

$$
f(a, b) \leq \frac{1}{A(D)} \iint_{D} f(x, y) d x d y \leq \frac{\sum_{k=1}^{n} f\left(a_{k}, b_{k}\right)}{n}
$$

where $(a, b)$ is the centre of $D,\left(a_{k}, b_{k}\right)(k=1,2, \ldots, n)$ are the vertices and $A(D)$ is the area of $D$.

Proof. Without loss of generality, we may assume that the centre $(a, b)$ is $(0,0)$, since otherwise we only need to do a translation. Note that $D$ being a regular $n$-gon 
implies that the image of $D$ under the rotation of an angle $\frac{2 k \pi}{n}$ with respect to the origin is still $D$ for any integer $k: k=0,1,2,3, \ldots, n-1$. For the sake of simplicity, we set

$$
\alpha_{k}=\cos \frac{2 k \pi}{n} \quad \text { and } \quad \beta_{k}=\sin \frac{2 k \pi}{n} .
$$

A rotation is an affine transformation. The jacobian is 1 . Hence

$$
\iint_{D} f(x, y) d x d y=\iint_{D} f\left(\alpha_{k} x-\beta_{k} y, \beta_{k} x+\alpha_{k} y\right) d x d y .
$$

It follows from (16) that

$$
\begin{aligned}
\iint_{D} f(x, y) d x d y & =\iint_{D} \frac{1}{n} \sum_{k=0}^{n-1} f\left(\alpha_{k} x-\beta_{k} y, \beta_{k} x+\alpha_{k} y\right) d x d y \\
& \geq \iint_{D} f\left(\frac{1}{n} \sum_{k=0}^{n-1}\left(\alpha_{k} x-\beta_{k} y\right), \frac{1}{n} \sum_{k=0}^{n-1}\left(\beta_{k} x+\alpha_{k} y\right)\right) d x d y \\
& =\iint_{D} f(0,0) d x d y \\
& =A(D) f(0,0),
\end{aligned}
$$

where the inequality holds because of Lemma 3.2, and the second equality because of Lemma 3.3. We thus prove the first part of (15).

To prove the second inequality of $(15)$, we draw $n$ segments between the centre $(0,0)$ and each vertex. Now $D$ is seperated by $n$ similar triangles and each has the area $\frac{A(D)}{n}$. On each triangle, we apply Theorem 2.4 and we have $n$ inequalities. The left side of each inequality is a product of $\frac{n}{A(D)}$ and an integral of $f$ over a triangle and the right side is one third of a sum of the values of $f$ at two vertices and at $(0,0)$. We add all $n$ integrals in the left side and all the terms in the right side. We have

$$
\frac{n}{A(D)} \iint_{D} f(x, y) d x d y \leq \frac{n f(0,0)+2 \sum_{k=1}^{n} f\left(a_{k}, b_{k}\right)}{3} .
$$

But by the first inequality of (15)

$$
f(0,0) \leq \frac{1}{A(D)} \iint_{D} f(x, y) d x d y
$$

and the above inequality, we have then

$$
\frac{2 n}{3 A(D)} \iint_{D} f(x, y) d x d y \leq \frac{2 \sum_{k=1}^{n} f\left(a_{k}, b_{k}\right)}{3} .
$$


Dividing both sides by $\frac{2 n}{3}$ gives the second part of (15).

If $n$ is larger and larger, the polygons become closer and closer to a disk. We have thus the following Hadamard's inequality on a disk. This inequality can be found also in [2]. For an improved inequality, see [1].

Theorem 3.5. If $f$ is a convex function on a disk $D$ centred at $(a, b)$, then

$$
f(0,0) \leq \frac{1}{A(D)} \iint_{D} f(x, y) d x d y \leq \frac{1}{l(C)} \int_{C} f(x, y) d s
$$

where $C$ is the boundary of $D$ and $l(C)$ the length of $C$.

Proof. We may assume that the centre of the disk is $(0,0)$. Let the radius of $D$ be $R$. Then $D=\left\{(x, y): x^{2}+y^{2} \leq R^{2}\right\}$ and $C=\left\{(x, y): x^{2}+y^{2}=R^{2}\right\}$. Let $D_{n}$ be a regular $n$-gon embedded in $D$. Then by (15), we have

$$
f(a, b) \leq \frac{1}{A\left(D_{n}\right)} \iint_{D_{n}} f(x, y) d x d y \leq \frac{\sum_{k=1}^{n} f\left(a_{k}, b_{k}\right)}{n} .
$$

Let $x=R \cos \theta, y=R \sin \theta$, then

$$
\begin{aligned}
\frac{\sum_{k=1}^{n} f\left(a_{k}, b_{k}\right)}{n} & =\frac{1}{2 \pi} \sum_{k=1}^{n} f\left(R \cos \theta_{k}, R \sin \theta_{k}\right) \frac{2 \pi}{n} \\
& \rightarrow \frac{1}{2 \pi} \int_{0}^{2 \pi} f(R \cos \theta, R \sin \theta) d \theta \quad(\text { if } n \rightarrow \infty) \\
& =\frac{1}{2 \pi R} \int_{C} f(x, y) d s .
\end{aligned}
$$

It is easy to see that $A\left(D_{n}\right) \rightarrow A(D)$ and $\iint_{D_{n}} f(x, y) d x d y \rightarrow \iint_{D} f(x, y) d x d y$ as $n \rightarrow \infty$. Therefore letting $n \rightarrow \infty$ in (18), we have (17).

\section{References}

[1] Y. Chen, Hadamard's inequality on a ring, preprint

[2] S. S. Dragomir, On Hadamard's inequality on a disk, JIPAM. J. Inequal. Pure Appl. Math. 1(2000), Article 2, 11.

[3] A. W. Robert and D. E. Varberg, Convex Functions, Academic Press, New York, 1973.

Department of Mathematical Sciences, Lakehead University, Thunder Bay, Ontario, Canada, P7B 5E1.

E-mail: yin.chen@lakeheadu.ca 